\title{
CATHEPSIN G DEGRADES SYNOVIAL FLUID LUBRICIN: RELEVANCE FOR OSTEOARTHRITIS PATHOGENESIS
}

Shan Huang ${ }^{1,+}$, Kristina A. Thomsson ${ }^{1,+}$, Chunsheng $\operatorname{Jin}^{1,2}$, Sally Alweddi ${ }^{1}$, André Struglics ${ }^{3}$, Ola Rolfson ${ }^{4}$, Lena I Björkman ${ }^{5}$, Sebastian Kalamajski ${ }^{6}$, Tannin A. Schmidt ${ }^{7}$, Gregory D. Jay ${ }^{8}$, Roman Krawetz ${ }^{9,10}$, Niclas G. Karlsson ${ }^{1,++,}$ and Thomas Eisler ${ }^{11,++}$

${ }^{1}$ Department of Medical Biochemistry and Cell Biology, Institute of Biomedicine, Sahlgrenska Academy, University of Gothenburg, Gothenburg, Sweden.

${ }^{2}$ Science for Life Laboratory, Department of Oncology-Pathology, Clinical Proteomics Mass Spectrometry, Karolinska Institutet, Solna, Sweden

${ }^{3}$ Department of Clinical Sciences Lund, Orthopaedics, Faculty of Medicine, Lund University, Lund, Sweden.

${ }^{4}$ Department of Orthopaedics, Institute of Clinical Sciences, The Sahlgrenska Academy, University of Gothenburg, Gothenburg, Sweden.

${ }^{5}$ Department of Rheumatology and Inflammation Research, Institute of Medicine, Sahlgrenska Academy, University of Gothenburg, Gothenburg, Sweden.

${ }^{6}$ Department of Molecular Skeletal Biology, Lund University, Lund, Sweden.

${ }^{7}$ Biomedical Engineering Department, University of Connecticut Health Center, Farmington, CT, USA.

${ }^{8}$ Department of Emergency Medicine, Warren Alpert Medical School and Division of Biomedical Engineering, School of Engineering, Brown University, Providence, Rhode Island, U.S.A.

${ }^{9}$ Cell Biology and Anatomy, Cumming School of Medicine, University of Calgary, 3330 Hospital Drive NW, Calgary, Alberta T2N4N1, Canada.

${ }^{10} \mathrm{McC}$ aig institute for Bone and Joint Health, University of Calgary, Calgary, Alberta T2N4N1, Canada.

${ }^{11}$ Department of Clinical Sciences, Danderyd Hospital, Karolinska Institutet, Stockholm, Sweden.

+ These authors have contributed equally to this work.

${ }^{++}$These authors have jointly supervised this work.

${ }^{*}$ Corresponding author - Niclas G. Karlsson, Department of Medical Biochemistry and Cell Biology, Institute of Biomedicine, Sahlgrenska Academy, University of Gothenburg, Sweden. Medicinaregatan 9A, 40530 Gothenburg, Sweden. Phone: +46 31 7866528; fax: +46 31 7862150; email: niclas.karlsson@medkem.gu.se 


\begin{abstract}
Lubricin (PRG4) is a mucin type protein that plays an important role in maintaining normal joint function by providing lubrication and chondroprotection. Improper lubricin modification and degradation has been observed in idiopathic osteoarthritis (OA), while the detailed mechanism still remains unknown. We hypothesized that the protease cathepsin $G$ (CG) may participate in degrading lubricin in synovial fluid (SF). The presence of endogenous CG in SF was confirmed in 16 patients with knee OA. Recombinant human lubricin (rhPRG4) and native lubricin purified from the SF of patients were incubated with exogenous CG and lubricin degradation was monitored using western blot, staining by Coomassie or Periodic Acid-Schiff in gels, and with proteomics. Full length lubricin ( $300 \mathrm{kDa})$, was efficiently digested with CG generating a 25-kDa protein fragment, originating from the densely glycosylated mucin domain ( 250 kDa). The 25-kDa fragment was present in the SF from OA patients, and the amount was increased after incubation with CG. A CG digest of rhPRG4 revealed 135 peptides and 72 glycopeptides, and confirmed that the protease could cleave in different domains of lubricin. Our results suggest that synovial CG may take part in the degradation of lubricin, which could affect the lubrication of OA joints.
\end{abstract}




\section{Introduction}

Osteoarthritis $(\mathrm{OA})$ is the most common degenerative joint disease, which affects people worldwide ${ }^{1}$. OA involves a progressive destruction of cartilage, bone and ligaments, sometimes causing extensive pain, reduced joint flexibility and may lead to severely impaired quality of life and rising health care costs. Recent research emphasises idiopathic $\mathrm{OA}$ as a multifactorial joint disease ${ }^{2}$ with an increasing number of studies demonstrating a low-grade inflammation with an accumulation of pro-inflammatory cytokines in synovial fluid (SF), which is associated with the pathological process of $\mathrm{OA}^{3}$. Cytokine triggered enzymatic degradation of matrix proteins is believed to contribute to the cartilage erosion found in $\mathrm{OA}^{4-7}$.

Lubricin, also known as Proteoglycan 4 (PRG4) is a large ( $300 \mathrm{kDa})$ extensively O-linked glycosylated mucinous protein in SF, and plays a critical role in maintaining cartilage integrity by providing boundary lubrication and reducing friction at the cartilage surface ${ }^{8}$. Besides the principle function of lubrication, lubricin also has growth-regulating properties ${ }^{9}$, prevents cell adhesion, provides chondroprotection ${ }^{10-12}$, and plays a role in the maturation of the subchondral bone ${ }^{13}$. Lubricin is predominantly synthesised and expressed by superficial zone chondrocytes at the cartilage surface layer ${ }^{14}$, but can also be secreted into the SF by synovial fibroblasts ${ }^{15}$ and stromal cells from peri-articular adipose tissues ${ }^{16}$. More recently it was reported that other sites and tissues, such as tendons, liver, kidney, skeletal muscles, and the ocular surface, also express lubricin ${ }^{17-19}$.

In the joint, lubricin is an extended molecule existing both as monomers and ashigher molecular mass complexes ${ }^{18}$. Lubricin has an approximately equal mass proportion of protein and oligosaccharides, and contains one central mucin-like region, two somatomedin B homology domains (SMB) in the $N$-terminal, one heparin or chondroitin sulfate binding domain, and one hemopexin-like domain (PEX) ${ }^{17,20}$ in the $C$-terminal (Fig. 1). The multiple domain structure contributes to diverse biological roles of lubricin. The $O$-linked oligosaccharides in the mucin domain render lubricin a low friction brush-like construct with repulsive hydration forces, provides lubrication during boundary movement, and makes lubricin water-soluble in $\mathrm{SF}^{21}$. The oligosaccharides are linked to abundant Thr/Ser residues, which are found in the repeat region, consisting of repeats with minor variations of the amino acid sequence 'EPAPTTPK', as well as its flanking, non-repeating Thr/Ser rich regions. The sparingly glycosylated terminal regions SMB and PEX domains have in other proteins been demonstrated to regulate innate immune processes by interacting with both complement and coagulation factors ${ }^{22}$ and assisting matrix protein binding ${ }^{23}$.

For over a decade, researchers have demonstrated that lubricin plays a vital role in inflammatory joint disease. Prevention of lubricin degradation may serve as a therapy in an early stage inflammatory arthritis ${ }^{24-28}$. Altered lubricin structure and change of lubricin concentrations which disrupts cartilage boundary lubrication is found in SF of OA patients ${ }^{29}$. As such, lubricin degradation may play a role in OA associated inflammation.

A limited number of proteases have been identified to degrade lubricin, among the more studied are lysosomal cysteine proteases cathepsin B, S, L and neutrophil elastase ${ }^{26,30,31}$. Cathepsin $G(C G)$ is one of the major neutrophil serine proteases that is synthesised in bone marrow and subsequently stored in the azurophil granules of polymorphonuclear neutrophils ${ }^{32,33}$. Upon activation of the granulocytes, CG is released at sites of inflammation and plays a crucial part in degrading chemokines and extracellular matrix (ECM) proteins, as well as regulating and activating pro-inflammatory cytokines ${ }^{34}$. Therefore, CG is reported to 
be active in various chronic inflammatory diseases ${ }^{35}$ and has for instance been shown to be highly active in the SF of rheumatoid arthritis patients.

Despite evidence indicating that lubricin modification contributes to some extent to OA initiation and pathological development, research on and understanding of enzymatic degradation of lubricin in OA is still limited. Hence, in this project, the serine protease CG was investigated for its participation in lubricin degradation in relation to $O A$. The presence of endogenous CG was detected in SF samples from 16 patients with idiopathic knee OA. The potent lubricin degrading ability of CG was confirmed both in vitro and in vivo, and the CG generated fragments of recombinant lubricin were identified.

\section{Results}

\section{Identification of CG in synovial fluid of OA subjects}

CG has previously been shown to be present in OA SF, however in a low abundance ${ }^{36}$. Since we hypothesized that CG plays a role in the OA pathology, we verified in our SF sample collection that CG was present. SF samples were collected from 16 late-stage OA patients and analysed for the presence of CG using SDS-PAGE and western blot with a polyclonal antiCG antibody. We could detect CG in all the SF samples at varying concentrations, the results are presented relative to a reference sample which was analysed on the same gel (Fig. 2a,b and Supplementary Fig. S1). Our results revealed that the CG levels in OA SF were estimated to be in the low ng range (1-10 ng) per $\mu \mathrm{SF}$. This is in line with a previous study using a general colorimetric substrate assay for trypsin/chymotryptic-like proteases in OA and RA $\mathrm{SF}^{36}$.

\section{CG degrades recombinant and native lubricin}

In order to investigate if CG was able to digest lubricin, we performed incubations using recombinant lubricin (rhPRG4), the degradation products were separated with SDS-PAGE and detected with lubricin mAb 9G3. This antibody targets the glycosylated mucin domain ${ }^{19}$. The results indicated that rhPRG4 was indeed degraded after extended incubation with only one major degradation product detected at approximately $25 \mathrm{kDa}$ (Fig. 3a).

To investigate if there was a difference in the ability to digest two different glycoforms of lubricin, we monitored the effect of CG digestion of both rhPRG4 and native lubricin using a Coomassie protein staining.Both rhPRG4 and native lubricin purified from SF were found to be degraded in a dose-dependent manner. No major degradation products were observed, and the degradation increased with prolonged incubation time (Figs $3 b, c$ and

Supplementary Figs S2, S3). rhPRG4 was found to be efficiently degraded by CG already after 30 minutes of incubation, illustrated by that $32 \%, 54 \%$ and $84 \%$ of the full length protein had been degraded, with increasing CG-to-protein ratio. After 16 hours, rhPRG4 was completely degraded (Fig. 3b). Native lubricin was more resilient to CG digestion. After 16 hours of incubation with the higher CG concentrations ( 0.5 and $2.5 \mathrm{w} / \mathrm{w} \% \mathrm{CG}$ ), 30 and $21 \%$ of the full length lubricin remained undigested, respectively. (Fig. 3c). These results suggested that the protein core of native lubricin was less accessible for CG digestion. We also showed that the effect of CG degradation of purified SF lubricin could be totally abolished using CG inhibitors (Supplementary Fig. S4). In all, we concluded that CG was capable of degrading lubricin both in its recombinant and native form. 


\section{CG degrades lubricin mucin domains in the presence of SF}

The decrease in Coomassie stain of lubricin after incubation with CG (Figs 3b, c and Supplementary Figs S2, S3) indicated that the protein backbone was substantially effected. We were interested to see if CG also could hydrolyse peptide bonds within the highly glycosylated mucin domains. Mucin domains are renowned for being hard to digest with proteolytic enzymes. Recombinant (rhPRG4) and purified lubricin from SF were incubated for two hours with CG, followed by separation on gels and developed with Periodic Schiff's base stain. This stains the glycans on the heavily glycosylated mucin domain of lubricin. Only two glycosylated degradation products (15- and 25-kDa) were detected on the SDS- gels (Fig. 4a), indicating that additional proteolytic products were of lower mass. The result supported previous observations, where both the glycosylated and non-glycosylated regions of lubricin were proteolytically digested by CG.

The influence of other components present in SF on CG degradation of the lubricin mucin domain was also assessed. Eight OA SF samples were incubated with or without supplement of exogenous CG. Lubricin was quantified using ELISA with mAb $9 G 3$ as the catching antibody and PNA lectin for detection. Peanut agglutinin lectin (PNA) binds to terminal galactose residues that are abundantly expressed on lubricin ${ }^{17}$. With this ELISA method, we could estimate the amounts of lubricin in OA SF to be in the range of 300-550 $\mathrm{gg} / \mathrm{ml}$ (Fig. 4b). After incubation with CG (8-15 w/w\%, enzyme to lubricin weight ratio), the amount of lubricin was decreased approximately $50 \%$. This experiment highlights that lubricin can be degraded in SF by an exogenous supply of CG. CG degradation of lubricin was less efficient in the presence of SF, compared to when performed in PBS (Fig 4a and Supplementary Fig. S5).

CG degradation of the lubricin mucin domain in SF was also visualized using the PNA lectin by western blots. After two hours of incubation of OA SF with CG, the intensity of the band corresponding to full length lubricin had decreased considerably compared to the control without CG, and the formation of a weakly stained band at $25 \mathrm{kDa}$ was observed (Fig. 4c). Western blot of SF incubated without CG revealed that in addition to full length lubricin, several other PNA reactive components of unknown origin at 50-100 kDa was detected. These components probably represent other PNA reactive proteins in SF as well as degradation products of lubricin.

\section{The 25-kDa lubricin fragment is found in SF from OA patients and is increased after CG incubation}

We were interested to investigate if the $25 \mathrm{kDa}$ mucin fragment generated from lubricin also could be found in SF, providing evidence that lubricin is degraded in its natural environment. Indeed, by increasing exposure time of the western blot using the mucin domain mAb 9G3, a single lubricin fragment at $25 \mathrm{kDa}$ of endogenous origin was observed in SF from $13 \mathrm{OA}$ patients without CG supplementation (Figs 5a,b and Supplementary Fig. S6). The selected region from a western blot shows the lubricin degradation fragment at $25 \mathrm{kDa}$ in SF from six patients (Fig. 5a).

We could also show that the amount of the $25 \mathrm{kDa}$ fragment was increased after addition of exogenous CG to SF (Fig. 5c and Supplementary Fig. S7). Complete degradation of full length lubricin was accompanied by a concomitant increase of a $25-k D a$ lubricin fragment, similar as 
was observed for rhPRG4 (Fig. 3a). We found that extending the incubation of lubricin with CG even for a longer period ( $>16$ hours, results not shown) eventually decreased the intensity of the 25-kDa fragment, indicating that CG was capable of digesting this component even further.

These experiments suggest that endogenous SF CG is at least partly responsible for generating the 25-kDa lubricin fragment found in OA patients.

\section{Proteomics and glycoproteomics analyses of rhPRG4 digested with CG}

In order to identify CG cleavage sites within lubricin, rhPRG4 was incubated with CG in PBS overnight at $37^{\circ} \mathrm{C}$ at a ratio of 1:45 (enzyme to substrate, by weight), and the obtained degradation products analysed with LC-MS/MS. We identified 135 non-glycosylated peptides and 72 glycopeptides in the size range of 6-37 amino acids, the majority from the $\mathrm{N}$ - and $\mathrm{C}$-terminal of lubricin (Supplementary Tables S1 and S2). CG has been described to have a combination of tryptic and chymotryptic type specificity, but is also reported to cleave at other amino acids ${ }^{37-39}$. An overview of the cleavage sites in lubricin detected here are displayed in Fig. 6a. As a control experiment, we performed semi-tryptic searches of tryptic digests of rhPRG4, which revealed that 13 peptides could originate from other sources than CG digestion, for example autoproteolytic degradation. The most frequent cleavage site was $\mathrm{C}$-terminal of lysine residues (33\%), a site which has been reported previously for $\mathrm{CG}^{37}$. Many peptides were found to be overlapping within the same regions in the peptide backbone. CG cleavage sites within the glycosylated mucin domain were identified after manual evaluation of MS spectra.

The glycopeptides constituted glycoforms made up of 35 peptide cores, carrying glycans of different monosaccharide compositions, and consisting of Hex, HexNAc and NeuAc residues, matching simple core 1 type $O$-glycosylation that is produced in $\mathrm{CHO}$ cells from where the rhPRG4 was expressed (Supplementary Table S2). Higher-energy collisional dissociation (HCD) fragmentation did not reveal glycan site specific information, however all but one deduced peptide contained potential $O$-glycan sites (Thr or Ser, and less common, Tyr). The peptide D.MDYLPRVPN.Q from the C-terminal (aa 1122-1130) was detected as two glycoforms and proposed to be glycosylated on a Tyr residue (Supplementary Fig. S8). The peptide L.RNGTVLAF.R (aa 1158-1165) was detected as two glycoforms, revealed monosaccharide compositions matching that of $\mathrm{N}$-glycans of high mannose type (Supplementary Fig. S9). We have also detected this potential $\mathrm{N}$-glycan site in tryptic digests (data not shown). Mass spectra of three glycopeptides with the peptide sequences 'ETAPTTPK', 'KEPAPTTPK' and 'KEPAPTTPKKPAPK' from within the mucin repeat region are shown in Fig $6 c$ and Supplementary Figs S10 and S11, respectively. These repeat peptides are found 3,17, and 2 times respectively in the mucin domain. Together with the identification of two nonglycosylated repeat peptides ('KEPAPTTPKKPAPK' and 'SAPTTTKEPAPTTTK' , Supplementary Table S1), these data provided mass spectrometric evidence for that CG may cleave within the mucin region. The $\mathrm{N}$-terminal derived glycopeptide with the sequence 'K.DNKKNRTKKKPTPKPPVVDEAG.S' (aa 202-223) contains two potential glycosylation sites (Thr208 and Thr213) and was detected as two glycoforms, where the monosaccharide compositions of the first glycoform supports the presence of a Tantigen (Galß1-3GalNAc), the second the sialylated T-antigen (Fig. 6b). CG was also able to cleave this glycopeptide stretch further (Supplementary Table S2). The CG cleavage sites within amino acid sequence 202-223 are summarized by vertical lines: 
DN $|K| K \mid$ NRTK $\mid$ KKPTPKPPVV|D|E $|A| G$. This example illustrates that CG can cleave after many different amino acids in lubricin, however at low efficiency. This promiscuity in cleavage site specificity has been highlighted in studies with defined substrates ${ }^{37}$.

\section{Discussion}

Despite being one of the most common and disabling joint diseases, OA pathogenesis and development are still largely unknown. The success of introducing recombinant lubricin for OA treatment in animal models ${ }^{40,41}$, indicate a role of lubricin in OA pathogenesis. To date, improper lubricin post-translational modifications, including glycosylation profile change ${ }^{42}$, forming complex with other matrix proteins ${ }^{43}$, and proteolytic cleavages are all reported in $\mathrm{OA}$. This may ultimately alter the surface lubrication and contribute to disease development.

Here we investigate the role of serine protease CG, with respect to its presence in SF as well as its degrading ability. CG has previously been suggested to be present in SF of OA-joints, using a colorimetric peptide substrate assay, specific for chymotryptic like proteases ${ }^{36}$. In this study, we validated the finding of CG in OA detecting endogenous CG in SF from 16 patients using western blot (Fig. 2).

A complete degradation of rhPRG4, even for the putatively protected mucin domain, was observed after CG incubation, and this digestion can be enhanced by a prolonged time and increased enzyme levels within physiologically relevant concentrations (Figs $3 a, b$ and Fig. 4a). Moreover, the degrading ability of CG was confirmed introducing CG inhibitor during incubation (Supplementary Fig. S4). These data showed that CG is an efficient protease capable of degrading rhPRG4. Inspired by these results we monitored the effect of CG digestion on native lubricin from SF from individual OA patients and from an RA pool. Similar degrading ability for native lubricin as with recombinant was achieved (Figs $3 c, 4 a$ ), and a near complete loss of full-length lubricin was observed after incubating with OA SF samples (Figs $4 b, 5 c$ ). The findings demonstrate the potent lubricin degrading ability of $C G$, both in a purified in vitro incubation system and in a more complex biological environment as in SF form OA patients. The lubricin degrading effect of CG was less pronounced when adding CG to lubricin in SF compared to purified lubricin (Supplementary Fig S5). Degrading efficiency may be influenced by differences in glycosylation of the two native lubricin variants (OA versus RA) ${ }^{42}$, and also for rhPRG4, the latter having $\mathrm{CHO}$ cell $O$-glycosylation. We hypothesize that the CG degrading efficiency in SF may also be affected by other SF located proteins competing with lubricin as CG substrates, or by serine protease inhibitors preventing CG activity.

The CG digests of both recombinant and native lubricin revealed a glycosylated lubricin degradation product of approximately $25 \mathrm{kDa}$, which was detected by western blot (using mAb $9 G 3$ or PNA) or with the glycosensitive stain PAS (Figs $3 a, 4 a, 4 c$, and $5 c$ ). The presence of glycosylation makes the fragment barely detectable with Coomassie protein stain, tryptic digests of the $25 \mathrm{kDa}$ fragment did not generate any peptides or glycopeptides, which may be expected due to dense and heterogeneous glycosylation, as well as MS ionization inefficiency. However, the data leaves little doubt that the $25 \mathrm{kDa}$ fragment in fact originates from the mucin repeat region, since it could be detected with the monoclonal lubricin antibody 9G3, which binds glycosylated forms of the sequence 'KEPAPTTT' ${ }^{19}$, present multiple times in the mucin domain.

With endogenous CG being present in SF of OA patients, we hypothesized that this protease could generate such a mucin domain containing fragment also in vivo. The detection of an 
endogenously produced 25 kDa lubricin fragment with mAb $9 G 3$ in SF from 13 late-stage OA patients (Fig 5a, b) confirmed that lubricin was indeed degraded partially in OA SF by a CG like protease. In all, our experiments thus supported that CG could be a protease candidate for proteolytic digestion of lubricin in SF. This reflects a local effect of CG during OA pathogenesis, which is in consistent with and extends previous findings that joint inflammation increases during $O A$ and plasma neutrophil activation can serve as a biomarker of $O A^{44}$. Our finding is in accordance with previous knowledge that $C G$ is notably released during inflammatory processes and contributes to ECM degradation ${ }^{34}$. CG as a traditional immune activator and regulator, in a recent research was proved to be responsible for proinflammatory IL-1 family cytokines activation ${ }^{45}$. Cytokine IL-1 $\beta$ is found to be active during $\mathrm{OA}$ and induces inflammatory responses that causes protease activation and degradation of ECM proteins ${ }^{46,47}$. Furthermore, decreased amount of lubricin was reported by treating cartilage with IL-1 $\beta$ in animal models ${ }^{28,48}$. The present study proves a lubricin degradation ability of $C G$, which renders $C G$ a potential considerable role during $O A$, strengthens the importance of further understanding of CG in OA disease and the connection between inflammation and lubricin modifications during the OA pathologic process.

CG has been reported to be a promiscuous protease with both chymase and tryptase activity capable of cleaving both at charged amino acids (eg. Lys and Arg) as well as hydrophobic amino acids (eg Phe, Trp, and Leu ) 37,39 . Proteomics and glycoproteomics of a CG digest of recombinant lubricin proved the enzyme to be an efficient protease, and gave an explanation for the degradation of lubricin observed in our western blots. We could detect peptides in the size range of 6-37 amino acids from both the $N$ - and $C$-terminal, as well as the mucin domain, proving that $\mathrm{CG}$ digested lubricin at numerous sites in the protein backbone. $33 \%$ of the identified peptides contained $C$-terminal Lys residues (Supplementary Table S1 and S2). Hence, the Lys residues which are frequently found throughout the lubricin repeat region in the mucin domain must be obvious targets for digestion. Lys is the most common amino acid (165 aa of total of 1404) in lubricin after Thr and Pro, the two main constituents of the mucin domain. We detected three glycopeptide sequences originating from the mucin domain (Supplementary Table S2). Limited peptide coverage within the mucin domain is expected, since glycosylated peptides from the mucin domain are often more difficult to detect (high heterogeneity, poor ionization efficiency etc). These three glycopeptides occur multiple times in the mucin domain. Together with the detection of two nonglycosylated peptides from the same region (Supplementary Table S1), they provide ample evidence for that the mucin domain, which with glycosylation has an estimated size of $250 \mathrm{kDa}$ (Fig 1), can be degraded into $25-\mathrm{kDa}$ or smaller fragments, as observed with western blot and proteomics.

In order to validate some of the lubricin peptides proposed to be formed by CG digestion in this study, we investigated the presence of 'in-vitro formed' autoproteolytic peptides in tryptic digests of recombinant lubricin (rhPRG4). Surprisingly, we did detect a small number of semitryptic peptides, which indicates that larger tryptic lubricin peptides may be degraded in the test tube to smaller semitryptic peptides that mistakenly can be assigned as a protease products. One example was the peptide H.VFMPEVTPDMDYLPR.V (aa1113-1127). The non-tryptic cleavage site at aa 1112 has previously been reported to be a cathepsin $\mathrm{S}$ cleavage site in tear fluid ${ }^{30}$, and equivalent site reported to be an endogenous protease cleavages in SF derived lubricin from horse ${ }^{44}$. Our data suggests an alternative explanation of post-proteolysis induced after tryptic digestion. 
$\mathrm{OA}$ is a multifactorial disease, and beyond all doubt there is more than one factor that contributes to OA lubricin modifications. The present work demonstrates the potency of CG for lubricin degradation, providing the hypothesis that $C G$ is involved and contributes to $O A$ disease development. The proteolytic cleavage product identified here has a potential to serve as a future biomarker for lubricin degradation in OA.

\section{Materials and methods}

\section{Lubricin and synovial fluid samples}

Synovial fluid (SF) samples were collected from 16 late-stage idiopathic OA patients (8 males and 8 females) subjected to knee replacement surgery. The mean age of the patients was 71 years (range 62-87 years). All individuals gave written consent and the procedure was approved by the ethics committee at Sahlgrenska University Hospital (ethical application 17215). The SF samples were collected prior surgery, centrifuged, aliquoted and stored at $-80^{\circ} \mathrm{C}$ until assayed. Recombinant lubricin (rhPRG4, $1 \mathrm{mg} / \mathrm{mL}$ in Phosphate Buffered Saline (PBS) + $0.1 \%$ Tween 20) was obtained from Lubris BioPharma, USA. CG (22 ng/ $\mu \mathrm{L}$ in PBS) was isolated from human leukocytes (Sigma-Aldrich, Germany). Purified native lubricin (SF Lub) was enriched from patients' synovial fluid (pooled from RA patients, $n=10$ ) by anion exchange chromatography and ethanol precipitation as described elsewhere ${ }^{49}$, and was quantified using lubricin sandwich ELISA as described below.

\section{In vitro digestion of lubricin by CG}

rhPRG4 and lubricin purified from SF, 2-5 $\mu$ g, were incubated with different concentrations (2.5-125 ng) of CG in at $37^{\circ} \mathrm{C}$ in PBS ( $\mathrm{pH} 7.4$ ) for 30 minutes or over-night (16 hours). SF (2$2.5 \mu \mathrm{L}$ ) were incubated with CG $(22-44 \mathrm{ng})$ in $37^{\circ} \mathrm{C}$ for 1 or 2 hours, or a time series of $30 \mathrm{~min}$ and 16 hours (specific digestion conditions are indicated in figures).

\section{SDS-PAGE}

Purified native lubricin and SF samples from CG incubations, and also SF samples (16 OA patient samples, $2 \mu \mathrm{lSF}$ ) used for screening of endogenous CG or lubricin degradation fragments, were reduced with $50 \mathrm{mM}$ dithiothreitol (DTT) (Merck KGaA) followed by boiling at $95^{\circ} \mathrm{C}$ for 15 minutes, and alkylation by $125 \mathrm{mM}$ iodoacetamide (Sigma-Aldrich, St. Louis, MO, US) in dark for 45 minutes. Samples were analysed on NuPAGE Tris-acetate 3-8\% gel (Invitrogen, Thermo Fisher Scientific, Waltham, MA, US). One $\mu$ g of rhPRG4 or 22 ng CG (for endogenous CG assay) were included on each gel as controls. Molecular weight was compared to PageRuler ${ }^{\mathrm{TM}}$ Plus Prestained Protein Ladder (10 to $250 \mathrm{kDa}$, Thermo Fisher Scientific, USA). Gels were stained either with Coomassie brilliant blue R-250 (Bio-Rad Laboratories, Hercules, CA, US) or with Periodic Acid-Schiff (PAS) (Sigma-Aldrich) according to the manufacturers' instructions. Lubricin band intensities were plotted and peak values were calculated by ImageJ (ImageJ 1.50i, USA) ${ }^{50}$.

\section{Western blot antibody and lectin staining}

After electrophoresis, the gels were blotted to an Immobilon-P PVDF Membrane (Merck Millipore, Burlington, MA, US) using Trans-Blot ${ }^{\circledR}$ SD Semi-Dry Transfer Cell (Bio-Rad 
Laboratories) at $200 \mathrm{~mA}$ for 80 minutes. After blocking with $1 \%$ bovine serum albumin (BSA) (VWR, Radnor, PA, US), the membranes were probed with $1 \mu \mathrm{g} / \mathrm{mL} \mathrm{mAb} 9 \mathrm{G} 3$ against the glycosylated epitope 'KEPAPTTT' in the lubricin mucin domain (Merck KGaA, Darmstadt, Germany ${ }^{51}$ ) or polyclonal anti-CG antibody (Abcam, UK) $1 / 1000$ diluted in assay buffer ( $1 \%$ BSA in PBS-Tween) for detection of endogenous CG in SF, followed by Horseradish Peroxidase (HRP) conjugated goat anti-mouse IgG $(\mathrm{H}+\mathrm{L})$ highly cross-adsorbed secondary antibody (Invitrogen, USA) 1/4000 diluted in assay buffer. For the lectin staining assay, blots were first probed by $1 \mu \mathrm{g} / \mathrm{ml}$ biotinylated Peanut Agglutinin (PNA, Vector laboratories, USA) followed by $0.2 \mu \mathrm{g} / \mathrm{ml}$ HRP-streptavidin (Vector laboratories, USA). After incubations, membranes were stained by WesternBright ECL Spray (Advansta, USA) and visualized in a luminescent image analyser (LAS-4000 mini, Fujifilm, Japan). Band intensities were calculated by Image $1.50 \mathrm{i}^{50}$.

\section{Lubricin sandwich ELISA}

An in-house ELISA method was set-up and validated for measuring lubricin concentrations in SF. Monoclonal antibody $9 \mathrm{G} 3(1 \mu \mathrm{g} / \mathrm{mL}$ in PBS) was coated on 96 -well Nunc-Immuno maxisorp plates (Thermo Fisher Scientific) at $4^{\circ} \mathrm{C}$ over-night. After blocking with $3 \%$ BSA in PBS+0.05\% Tween, SF samples were added as a dilution series (1/50) in assay buffer $(1 \%$ BSA in PBS-Tween) and incubated for 1 hour at room temperature (RT). Bound proteins were then incubated with biotinylated PNA (Vector laboratories, CA, USA) $(1 \mu \mathrm{g} / \mathrm{mL}, 1$ hour at $\mathrm{RT}$ ), followed by HRP-streptavidin (Vector laboratories) at $0.1 \mu \mathrm{g} / \mathrm{mL}$ (1 hour at RT). Between each incubation, the wells were washed three times with PBS-Tween to remove unbound reagents. Proteins were stained with 1-Step ${ }^{\text {TM }}$ Ultra TMB-ELISA Substrate Solution (Thermo Fisher Scientific) until blue colour was fully generated and the reaction stopped by adding 1 $\mathrm{M} \mathrm{H}_{2} \mathrm{SO}_{4}$. Absorbances were read at $450 \mathrm{~nm}$, and compared with a standard curve using recombinant lubricin (dilution series of rhPRG4 $(1 \mathrm{mg} / \mathrm{mL}$ ) in assay buffer). Samples were measured in duplicates and mean values are reported. The lubricin ELISA had an intra plate $\mathrm{CV}=7.5 \%$ ( $\mathrm{n}=1 \mathrm{SF}$ sample with 10 repeats) and a inter plate $\mathrm{CV} \%=7.8 \%$ ( $\mathrm{n}=1 \mathrm{SF}$ sample, tested on 4 plates).

\section{LC-MS/MS and MS data analyses}

rhPRG4 (10 $\mu \mathrm{g}$ in $10 \mu \mathrm{l} \mathrm{PBS}+0.1 \%$ Tween 20) was incubated with CG $(0.22 \mu \mathrm{g}$ in $10 \mu \mathrm{L})$ in $37^{\circ} \mathrm{C}$ under non-reducing conditions over night. Incubations were performed with or without subsequent Tween removal (Pierce Detergent Removal Spin Column $125 \mu \mathrm{L}$, Thermo Fisher Scientific), but the latter approach generated fewer detected peptides and were therefore not reported here. For control experiments and semitryptic searches, rhPRG4 was digested with trypsin in-solution or in-gel as described elsewhere ${ }^{52}$. The peptides were desalted using C18 ziptips, followed by separation with LC-MS using in-house packed C18 columns at a flow rate of $200 \mathrm{~nL} / \mathrm{min}$, and a $45-\mathrm{min}$ gradient of $5-40 \%$ buffer B (A: $0.1 \%$ formic acid, B:0.1\% formic acid, $80 \%$ acetonitrile). The column was connected to an Easy-nLC 1000 system (Thermo Fisher Scientific, Odense, Denmark), a nano-electrospray ion source and a QExactive Hybrid Quadrupole-Orbitrap Mass Spectrometer (Thermo Fisher Scientific). For full scan MS, the instrument was scanned $\mathrm{m} / \mathrm{z}$ 350-2000, resolution 60000 ( $\mathrm{m} / \mathrm{z} 200)$, AGC target 3e6, max IT $20 \mathrm{~ms}$, dynamic exclusion $10 \mathrm{sec}$. The twelve most intense peaks (charge states $2,3,4$ ) were selected for fragmentation with higher-energy collisional dissociation (HCD). 
For MS/MS, resolution was set to 15000 ( $\mathrm{m} / \mathrm{z} 200)$, AGC target to $5 \mathrm{e} 5$, max IT $40 \mathrm{~ms}$, and collision energy NCE $=27 \%$.

Raw data files were searched against the human Uniprot protein database (downloaded 17.11.2017) using Peaks Studio 8.5 (Bioinformatics Solutions Inc., Waterloo, Canada). For peptide identification, mass precursor error tolerance was set to $5 \mathrm{ppm}$, and fragment mass error tolerance to $0.03 \mathrm{Da}$, enzyme: none; variable modifications: oxidation (M) and deamidation (NQ). Peptide-spectrum matches were filtered to $0.1 \%$ false discovery rate (Peaks peptide score $>25$ ). Glycopeptide MS-MS spectra were selected with Peaks Studio software and/or manually using glycan diagnostic ions in the lower mass range $(\mathrm{m} / \mathrm{z} 186$, $204,274,292,366)$. Spectra were evaluated manually, all major fragment ions were assigned and $>4 \mathrm{~b} / \mathrm{y}$ ions required in order to identify the peptide backbone. The assignments were aided by proteomics mining tools available free of charge (Findpept and PeptideMass (web.expasy.org); MS-product (Protein Prospector http://prospector.ucsf.edu). Mass precursor errors were less than $5 \mathrm{ppm}$.

\section{Acknowledgements}

This study was funded by grants for the Swedish state under the agreement between the Swedish government and the county council, the ALF-agreement (ALFGBG-722391), the Swedish Research Council (621-2013-5895), Kung Gustav V:s 80-års foundation, Petrus and Augusta Hedlund's foundation (M-2016-0353), AFA insurance research fund (dnr 150150) and IngaBritt and Arne Lundberg Foundation. Sofia Grindberg and Paula-Therese Kelly Pettersson at Danderyd's Hospital and Lotta Falkendahl at University of Gothenburg are acknowledged for their assistance in collecting samples. Lubris BioPharma, LLC for providing rhPRG4.

\section{Ethical approval}

All OA and RA patients gave informed consent and all the procedures were approved by the regional ethical review board in Gothenburg (172-15,13/5-2015). The study conformed to the ethical guidelines of the 1975 Declaration of Helsinki of research involving human subjects. All methods were performed in accordance with the relevant guidelines and regulations.

\section{Author contributions}

AS and SK suggested the idea and performed initial investigation of lubricin digestion; $\mathrm{SH}, \mathrm{SA}$ and KAT performed the CG experiments and analysed the data; $\mathrm{CJ}$ purified patient lubricin; TAS and GDJ provided rhPRG4; LIB, OR, RK and TE provided patient material. SH, KAT, and NGK wrote the initial manuscript draft; KAT, NGK and TE updated the manuscript. All authors discussed the results, commented, and approved the final manuscript.

\section{Competing interests}

GDJ, RK and TAS authored patents related to rhPRG4 and hold equity in Lubris BioPharma, LLC. TAS is also a paid consultant for Lubris BioPharma, LLC. SK, CJ and NGK authored a 
bioRxiv preprint doi: https://doi.org/10.1101/792184; this version posted October 15,2019 . The copyright holder for this preprint (which

was not certified by peer review) is the author/funder. All rights reserved. No reuse allowed without permission.

patent using lubricin for diagnostics. SH, KAT, SA AS, OR, LIB, RK and TE declare no competing interest. 


\section{References}

1 Vos, T. et al. Global, regional, and national incidence, prevalence, and years lived with disability for 310 diseases and injuries, 1990-2015: a systematic analysis for the Global Burden of Disease Study 2015. Lancet 388, 1545-1602, doi:10.1016/S0140-6736(16)31678-6 (2016).

2 Lee, A. S. et al. A current review of molecular mechanisms regarding osteoarthritis and pain. Gene 527, 440-447, doi:10.1016/j.gene.2013.05.069 (2013).

3 Robinson, W. H. et al. Low-grade inflammation as a key mediator of the pathogenesis of osteoarthritis. Nature reviews. Rheumatology 12, 580-592, doi:10.1038/nrrheum.2016.136 (2016).

4 Morris, E. A., McDonald, B. S., Webb, A. C. \& Rosenwasser, L. J. Identification of interleukin-1 in equine osteoarthritic joint effusions. Am J Vet Res 51, 59-64 (1990).

5 Alwan, W. H. et al. Interleukin-1-like activity in synovial fluids and sera of horses with arthritis. Res Vet Sci 51, 72-77 (1991).

6 Goldring, M. B. Anticytokine therapy for osteoarthritis. Expert Opin Biol Ther 1, 817829, doi:10.1517/14712598.1.5.817 (2001).

7 Kamm, J. L., Nixon, A. J. \& Witte, T. H. Cytokine and catabolic enzyme expression in synovium, synovial fluid and articular cartilage of naturally osteoarthritic equine carpi. Equine Vet J 42, 693-699, doi:10.1111/j.2042-3306.2010.00140.x (2010).

8 Jay, G. D. et al. Association between friction and wear in diarthrodial joints lacking lubricin. Arthritis Rheum 56, 3662-3669, doi:10.1002/art.22974 (2007).

9 Marcelino, J. et al. CACP, encoding a secreted proteoglycan, is mutated in camptodactyly-arthropathy-coxa vara-pericarditis syndrome. Nat Genet 23, 319-322 (1999).

10 Wong, B. L., Kim, S. H., Antonacci, J. M., Mcllwraith, C. W. \& Sah, R. L. Cartilage shear dynamics during tibio-femoral articulation: effect of acute joint injury and tribosupplementation on synovial fluid lubrication. Osteoarthritis Cartilage 18, 464-471, doi:10.1016/j.joca.2009.11.008 (2010).

11 Waller, K. A. et al. Role of lubricin and boundary lubrication in the prevention of chondrocyte apoptosis. Proc Natl Acad Sci $U S A$ A 110, 5852-5857, doi:10.1073/pnas.1219289110 (2013).

12 D'Lima, D. D., Hashimoto, S., Chen, P. C., Colwell, C. W., Jr. \& Lotz, M. K. Human chondrocyte apoptosis in response to mechanical injury. Osteoarthritis Cartilage 9, 712-719, doi:10.1053/joca.2001.0468 (2001).

13 Abubacker, S. et al. Absence of proteoglycan 4 (Prg4) leads to increased subchondral bone porosity which can be mitigated through intra-articular injection of PRG4. J Orthop Res, doi:10.1002/jor.24378 (2019).

14 Flannery, C. R. et al. Articular cartilage superficial zone protein (SZP) is homologous to megakaryocyte stimulating factor precursor and Is a multifunctional proteoglycan with potential growth-promoting, cytoprotective, and lubricating properties in cartilage metabolism. Biochem Biophys Res Commun 254, 535-541, doi:10.1006/bbrc.1998.0104 (1999). 
15 Jay, G. D., Tantravahi, U., Britt, D. E., Barrach, H. J. \& Cha, C. J. Homology of lubricin and superficial zone protein (SZP): products of megakaryocyte stimulating factor (MSF) gene expression by human synovial fibroblasts and articular chondrocytes localized to chromosome 1q25. J Orthop Res 19, 677-687, doi:10.1016/S0736-0266(00)00040-1 (2001).

16 Lee, S. Y., Nakagawa, T. \& Reddi, A. H. Induction of chondrogenesis and expression of superficial zone protein (SZP)/lubricin by mesenchymal progenitors in the infrapatellar fat pad of the knee joint treated with TGF-beta1 and BMP-7. Biochem Biophys Res Commun 376, 148153, doi:10.1016/j.bbrc.2008.08.138 (2008).

17 Jay, G. D., Harris, D. A. \& Cha, C. J. Boundary lubrication by lubricin is mediated by Olinked beta(1-3)Gal-GalNAc oligosaccharides. Glycoconjugate J 18, 807-815, doi: 10.1023/A:1021159619373 (2001).

18 Jay, G. D. \& Waller, K. A. The biology of lubricin: near frictionless joint motion. Matrix Biol 39, 17-24, doi:10.1016/j.matbio.2014.08.008 (2014).

$19 \mathrm{Ai}, \mathrm{M}$. et al. Anti-Lubricin Monoclonal Antibodies Created Using Lubricin-Knockout Mice Immunodetect Lubricin in Several Species and in Patients with Healthy and Diseased Joints. Plos One 10, doi:10.1371/journal.pone.0116237 (2015).

20 Swann, D. A., Silver, F. H., Slayter, H. S., Stafford, W. \& Shore, E. The molecular structure and lubricating activity of lubricin isolated from bovine and human synovial fluids. Biochem J 225, 195-201 (1985).

21 Jay, G. D. Characterization of a bovine synovial fluid lubricating factor. I. Chemical, surface activity and lubricating properties. Connect Tissue Res 28, 71-88 (1992).

22 Deng, G., Curriden, S. A., Hu, G., Czekay, R. P. \& Loskutoff, D. J. Plasminogen activator inhibitor-1 regulates cell adhesion by binding to the somatomedin $B$ domain of vitronectin. $J$ Cell Physiol 189, 23-33, doi:10.1002/jcp.1133 (2001).

23 Piccard, H., Van den Steen, P. E. \& Opdenakker, G. Hemopexin domains as multifunctional liganding modules in matrix metalloproteinases and other proteins. J Leukoc Biol 81, 870-892, doi:10.1189/jlb.1006629 (2007).

24 Jay, G. D. et al. Lubricating ability of aspirated synovial fluid from emergency department patients with knee joint synovitis. J Rheumatol 31, 557-564 (2004).

25 Schmidt, T. A., Schumacher, B. L., Klein, T. J., Voegtline, M. S. \& Sah, R. L. Synthesis of proteoglycan 4 by chondrocyte subpopulations in cartilage explants, monolayer cultures, and resurfaced cartilage cultures. Arthritis Rheum 50, 2849-2857, doi:10.1002/art.20480 (2004).

26 Elsaid, K. A., Jay, G. D., Warman, M. L., Rhee, D. K. \& Chichester, C. O. Association of articular cartilage degradation and loss of boundary-lubricating ability of synovial fluid following injury and inflammatory arthritis. Arthritis Rheum 52, 1746-1755, doi:10.1002/art.21038 (2005).

27 Young, A. A. et al. Proteoglycan 4 downregulation in a sheep meniscectomy model of early osteoarthritis. Arthritis Res Ther 8, R41, doi:10.1186/ar1898 (2006).

28 Elsaid, K. A., Jay, G. D. \& Chichester, C. O. Reduced expression and proteolytic susceptibility of lubricin/superficial zone protein may explain early elevation in the coefficient of friction in the joints of rats with antigen-induced arthritis. Arthritis Rheum 56, 108-116, doi:10.1002/art.22321 (2007). 
29 Musumeci, G. et al. Lubricin expression in human osteoarthritic knee meniscus and synovial fluid: a morphological, immunohistochemical and biochemical study. Acta Histochem 116, 965-972, doi:10.1016/j.acthis.2014.03.011 (2014).

30 Elsaid, K. A., Jay, G. D. \& Chichester, C. O. Reduced expression and proteolytic susceptibility of lubricin/superficial zone protein may explain early elevation in the coefficient of friction in the joints of rats with antigen-induced arthritis. Arthritis Rheum 56, 108-116, doi:10.1002/art.22321 (2007).

31 Regmi, S. C. et al. Degradation of proteoglycan 4/lubricin by cathepsin S: Potential mechanism for diminished ocular surface lubrication in Sjogren's syndrome. Exp Eye Res 161, 1-9, doi:10.1016/j.exer.2017.05.006 (2017).

32 Senior, R. M. et al. Elastase of U-937 monocytelike cells. Comparisons with elastases derived from human monocytes and neutrophils and murine macrophagelike cells. $J$ Clin Invest 69, 384-393 (1982).

33 Campbell, E. J., Silverman, E. K. \& Campbell, M. A. Elastase and cathepsin G of human monocytes. Quantification of cellular content, release in response to stimuli, and heterogeneity in elastase-mediated proteolytic activity. J Immunol 143, 2961-2968 (1989).

34 Wiedow, O. \& Meyer-Hoffert, U. Neutrophil serine proteases: potential key regulators of cell signalling during inflammation. J Intern Med 257, 319-328, doi:10.1111/j.13652796.2005.01476.x (2005).

35 Korkmaz, B., Horwitz, M. S., Jenne, D. E. \& Gauthier, F. Neutrophil Elastase, Proteinase 3, and Cathepsin G as Therapeutic Targets in Human Diseases. Pharmacol Rev 62, 726-759, doi:10.1124/pr.110.002733 (2010).

36 Miyata, J. et al. Cathepsin G: the significance in rheumatoid arthritis as a monocyte chemoattractant. Rheumatology international 27, 375-382, doi:10.1007/s00296-006-0210-8 (2007).

37 Thorpe, M. et al. Extended cleavage specificity of human neutrophil cathepsin G: A low activity protease with dual chymase and tryptase-type specificities. PLoS One 13, e0195077, doi:10.1371/journal.pone.0195077 (2018).

38 Nguyen, M. T. N., Shema, G., Zahedi, R. P. \& Verhelst, S. H. L. Protease Specificity Profiling in a Pipet Tip Using "Charge-Synchronized" Proteome-Derived Peptide Libraries. J Proteome Res 17, 1923-1933, doi:10.1021/acs.jproteome.8b00004 (2018).

39 O'Donoghue, A. J. et al. Global substrate profiling of proteases in human neutrophil extracellular traps reveals consensus motif predominantly contributed by elastase. PLoS One 8, e75141, doi:10.1371/journal.pone.0075141 (2013).

40 Flannery, C. R. et al. Prevention of cartilage degeneration in a rat model of osteoarthritis by intraarticular treatment with recombinant lubricin. Arthritis Rheum 60, 840847, doi:10.1002/art.24304 (2009).

41 Waller, K. A. et al. Intra-articular Recombinant Human Proteoglycan 4 Mitigates Cartilage Damage After Destabilization of the Medial Meniscus in the Yucatan Minipig. The American journal of sports medicine 45, 1512-1521, doi:10.1177/0363546516686965 (2017). 
42 Estrella, R. P., Whitelock, J. M., Packer, N. H. \& Karlsson, N. G. The glycosylation of human synovial lubricin: implications for its role in inflammation. Biochem J 429, 359-367, doi:10.1042/BJ20100360 (2010).

43 Flowers, S. A. et al. Lubricin binds cartilage proteins, cartilage oligomeric matrix protein, fibronectin and collagen II at the cartilage surface. Sci Rep-Uk 7, doi:10.1038/s41598017-13558-y (2017).

44 Tasoglu, O., Boluk, H., Sahin Onat, S., Tasoglu, I. \& Ozgirgin, N. Is blood neutrophillymphocyte ratio an independent predictor of knee osteoarthritis severity? Clin Rheumatol 35, 1579-1583, doi:10.1007/s10067-016-3170-8 (2016).

45 Henry, C. M. et al. Neutrophil-Derived Proteases Escalate Inflammation through Activation of IL-36 Family Cytokines. Cell Rep 14, 708-722, doi:10.1016/j.celrep.2015.12.072 (2016).

46 Lianxu, C., Hongti, J. \& Changlong, Y. NF-kappaBp65-specific siRNA inhibits expression of genes of COX-2, NOS-2 and MMP-9 in rat IL-1beta-induced and TNF-alpha-induced chondrocytes. Osteoarthritis Cartilage 14, 367-376, doi:10.1016/j.joca.2005.10.009 (2006).

47 Goldring, M. B. Anticytokine therapy for osteoarthritis. Expert Opin Biol Th 1, 817-829, doi:Doi 10.1517/14712598.1.5.817 (2001).

48 Svala, E. et al. Characterisation of lubricin in synovial fluid from horses with osteoarthritis. Equine Vet J 49, 116-123, doi:10.1111/evj.12521 (2017).

49 Jin, C. et al. Human synovial lubricin expresses sialyl Lewis $x$ determinant and has Lselectin ligand activity. J Biol Chem 287, 35922-35933, doi:10.1074/jbc.M112.363119 (2012).

50 Schneider, C. A., Rasband, W. S. \& Eliceiri, K. W. NIH Image to ImageJ: 25 years of image analysis. Nat Methods 9, 671-675 (2012).

$51 \mathrm{Ai}, \mathrm{M}$. et al. Anti-lubricin monoclonal antibodies created using lubricin-knockout mice immunodetect lubricin in several species and in patients with healthy and diseased joints. PLoS One 10, e0116237, doi:10.1371/journal.pone.0116237 (2015).

52 Ali, L. et al. The O-glycomap of lubricin, a novel mucin responsible for joint lubrication, identified by site-specific glycopeptide analysis. Mol Cell Proteomics 13, 3396-3409, doi:10.1074/mcp.M114.040865 (2014).

53 Varki, A. et al. Symbol Nomenclature for Graphical Representations of Glycans. Glycobiology 25, 1323-1324, doi:10.1093/glycob/cwv091 (2015). 


\section{Figure legends}

Figure 1. (a) Description of lubricin and its domains. Lubricin contains a heavily glycosylated Pro/Thr rich mucin domain, flanked by two somatomedin B domains (SMB) and a hemopexin (PEX) domain. The $\mathrm{O}$-glycans are linked to Thr and Ser residues, and increases the size of the mucin domain from $86 \mathrm{kD}$ (without glycans) to approximately $250 \mathrm{kD}$. The monoclonal lubricin antibody $9 \mathrm{G} 3$ has been shown to bind the glycosylated peptide sequence 'KEPAPTTT' which is found eight times in the repeat region. Monosaccharides constituting lubricin oligosaccharides are represented by Symbol Nomenclature for Glycans (SNFG) ${ }^{53}$, where $\mathrm{N}$ acetylgalactosamine (GalNAc) is a yellow square, galactose (Gal) is a yellow circle and $N$ acetylneuraminic acid (NeuAc) is a purple diamond.

Figure 2. Endogenous Cathepsin G (CG) in synovial fluid (SF) from OA patients. (a) CG was detected in SF from 16 OA patients. SF was analysed with SDS-PAGE followed by western blot using a polyclonal anti-CG antibody. Bands corresponding to CG were detected at 29 $\mathrm{kDa}$, and the results are displayed as relative intensities (\% of absorbance), compared to a reference compound analysed on the same gel ( $22 \mathrm{ng}$ CG). Individual subject values from a selected region from the western blot showing the $29 \mathrm{kDa}$ CG bands from five patients (b) are highlighted in blue. The full-length western blot is displayed in Supplementary Fig. S1.

Figure 3. Cathepsin G (CG) degrades lubricin. rhPRG4 and purified lubricin from synovial fluid (SF Lub) were incubated with CG or without CG (control) at increasing enzyme to substrate ratios (weight/weight) in PBS for 30 min or 16 hours, followed by analyses on trisacetate gels (3-8\%). rhPRG4 was detected with western blot using mAb $9 G 3$ (a) or using Coomassie Blue stain (rhPRG4 and purified SF lubricin, Supplementary Figs S2 and S3). Coomassie Blue band intensities of full length lubricin incubated with CG compared to the control lubricin band (no CG) are displayed in (b) and (c) with the relative intensities in \% displayed as a number in each bar.

Figure 4. PAS and PNA lectin detection of lubricin and glycosylated lubricin fragments after incubation with Cathepsin G (CG). (a) Periodic Acid Schiff (PAS) staining of recombinant lubricin (rhPRG4) and purified lubricin from synovial fluid (SF LUB). rhPRG4 and SF LUB were incubated with $5 \mathrm{w} / \mathrm{w} \%$ CG (enzyme to substrate ratio by weight) in PBS for 2 hours, followed by analysis with SDS-PAGE and staining with PAS, which stains densely glycosylated proteins. (b) Lubricin quantification in SF using ELISA. SF $(2 \mu \mathrm{l})$ from eight OA patients were incubated with CG (44ng) or without CG for 2 hours at $37^{\circ} \mathrm{C}$. Lubricin was measured using a sandwich ELISA, with mAb $9 \mathrm{G} 3$ as the catching Ab and Peanut agglutinin (PNA) lectin for detection, and compared to a standard curve with rhPRG4. Data are presented as mean +/SEM. (c) PNA lectin western blot of SF incubated with CG. SF $(2.5 \mu \mathrm{L})$ from an OA patient was incubated with or without CG $(55 \mathrm{ng})$ for 2 hours at $37^{\circ} \mathrm{C}$.

Figure 5. Identification of the $25 \mathrm{kDa}$ glycosylated lubricin fragment from synovial fluid (SF) of OA patients. (a) Detection of an endogenous lubricin degradation fragment at $25 \mathrm{kDa}$ in SF from OA patients. SF samples $(2 \mu \mathrm{l})$ were analysed with SDS-PAGE, followed by western blot using $\mathrm{mAb} 9 \mathrm{G} 3$. Selected region from a western blot showing examples of a lubricin degradation fragment at $25 \mathrm{kDa}$ from six OA patients. The full-length western blot is displayed in Supplementary Fig. S6. (b) Semiquantification of the 25-kDa lubricin fragment in SF from 13 OA patients. The 25-kDa band intensities from western blot analyses are plotted relative to the full length lubricin band for every patient sample. rhPRG4 $(1 \mu \mathrm{g})$ was used as negative control. Individual subject values from the western blot in (a) are highlighted in blue. (c) SF incubated with exogenous CG. SF $(2 \mu \mathrm{L})$ from an OA patient was incubated with 
CG (44 ng) for 0 or 16 hours, followed by SDS-PAGE and detection with mAb 9G3. The original western blot is displayed in Supplementary Fig. $\mathrm{S7.}$

Figure 6. (a) Recombinant lubricin (rhPRG4) was digested with cathepsin G (CG), followed by analyses of the peptides with proteomics and glycoproteomics. Identified cleavage sites along the lubricin protein are represented by vertical lines. The complete peptide/glycopeptide lists are found in Supplementary Tables S1 and S2. (b) and (c) Higherenergy collisional dissociation (HCD) spectra of $O$-glycopeptides of rhPRG4 digested with CG, and analysed with LC/MS/MS. Diagnostic glycan oxonium ions are detected in the lower mass range ( $m / z$ 100-400). b/y-ions are detected without glycan substituents. Potential $O-$ glycan sites (Ser/Thr) are underlined and in bold. (b) The glycopeptide at $m / z 1035.2070(3+)$ eluting at $14.3 \mathrm{~min}$ was deduced to have the sequence 'DNKKNRTKKKPTPKPPVVDEAG' and carry the Sialyl T-antigen (NeuAc-Gal-GalNAc). The glycopeptide originates from the lubricin $\mathrm{N}$-terminal (aa positions 202-233). (c) The glycopeptide at $\mathrm{m} / \mathrm{z} 605.2905(2+)$ eluting at 15.015.4 min was deduced to have the sequence 'ETAPTTPK' and carry the T-antigen (GalGalNAc). The peptide originates from the mucin domain and is found three times in lubricin (aa positions 615-622,703-710, 825-832). 
Figure 1

N-terminal

Mucin domain

C-terminal

aa 1-231 (26 kDa) approx. 250 kDa

距

Pro-Thr-Thr-Lys-Glu-Pro-Ala-Pro-Thr-Thr-Pro-Lys

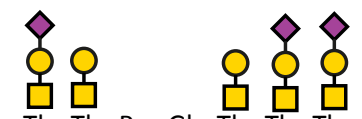

4

-Thr-Thr-Pro-Glu-Thr-Thr-Thr-

\begin{tabular}{|c|c|c|c|c|c|}
\hline $\begin{array}{l}\text { SMB } 1 \\
\text { SMB } 2\end{array}$ & $\begin{array}{c}\text { Non repeat } \\
\text { region }\end{array}$ & $\mathrm{MUClN}$ & Repeat region & $\begin{array}{c}\text { Non repeat } \\
\text { region }\end{array}$ & PEX \\
\hline
\end{tabular}

mAb $9 G 3$ recognition region 
Figure 2

a
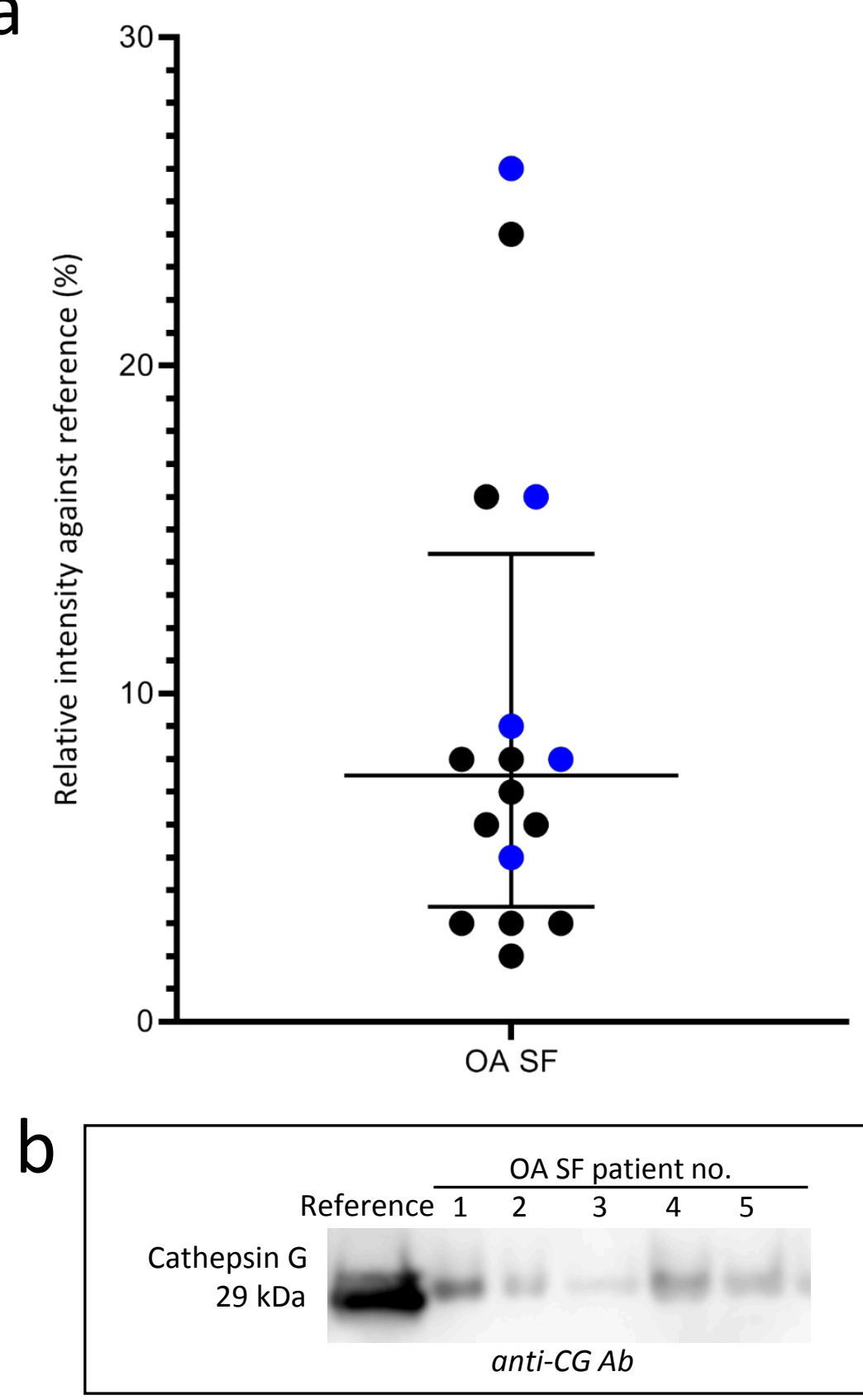
Figure 3

a

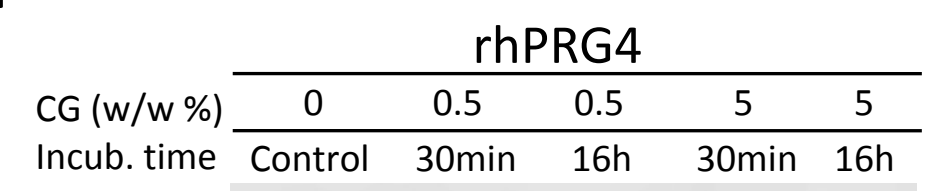

LUB

(> $250 \mathrm{kDa}$ )
25  b

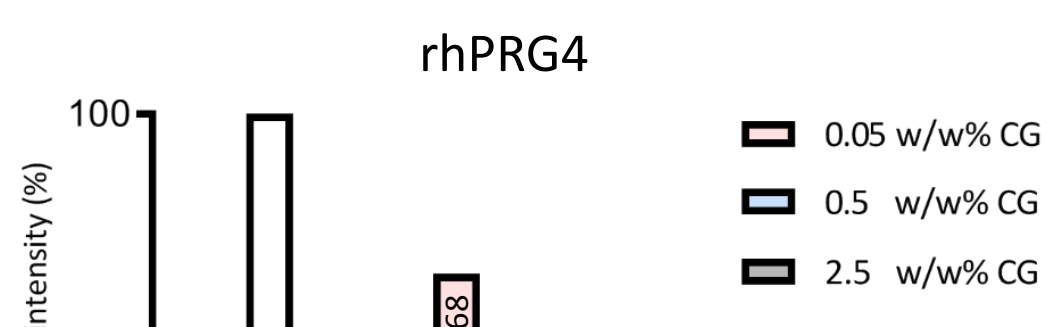

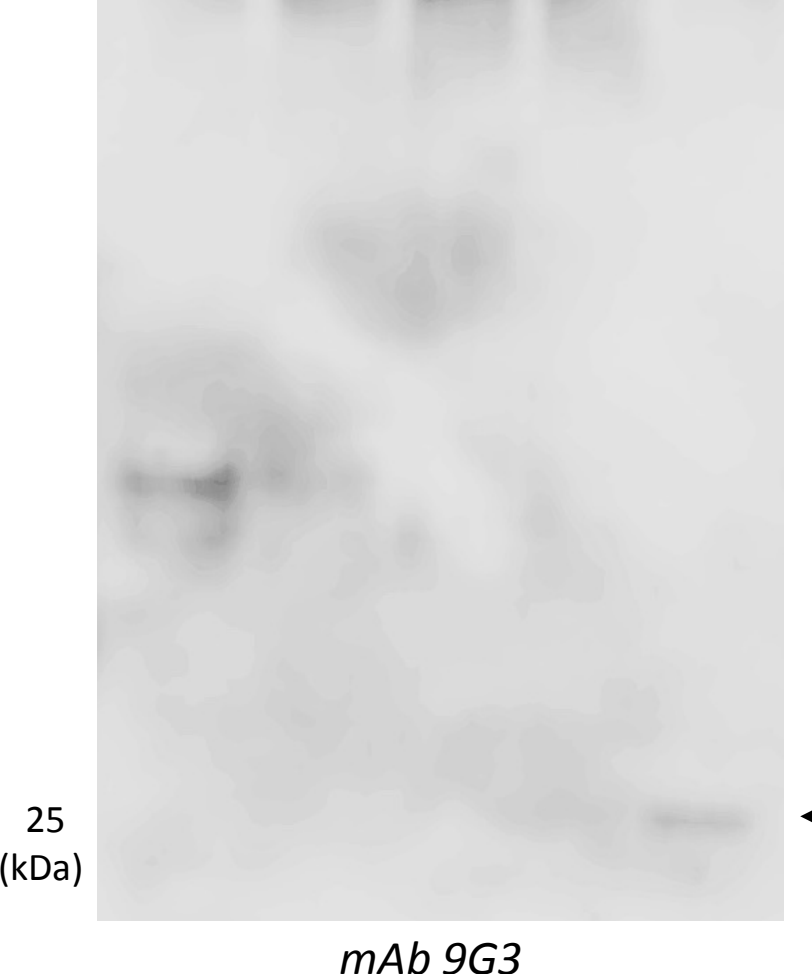

C

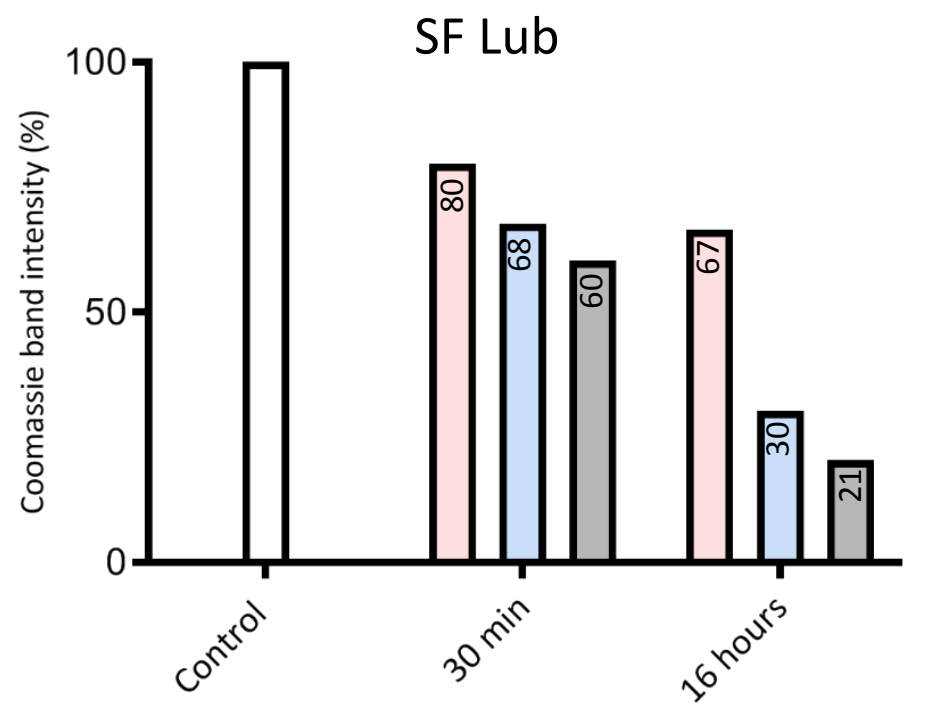




\section{Figure 4}

a
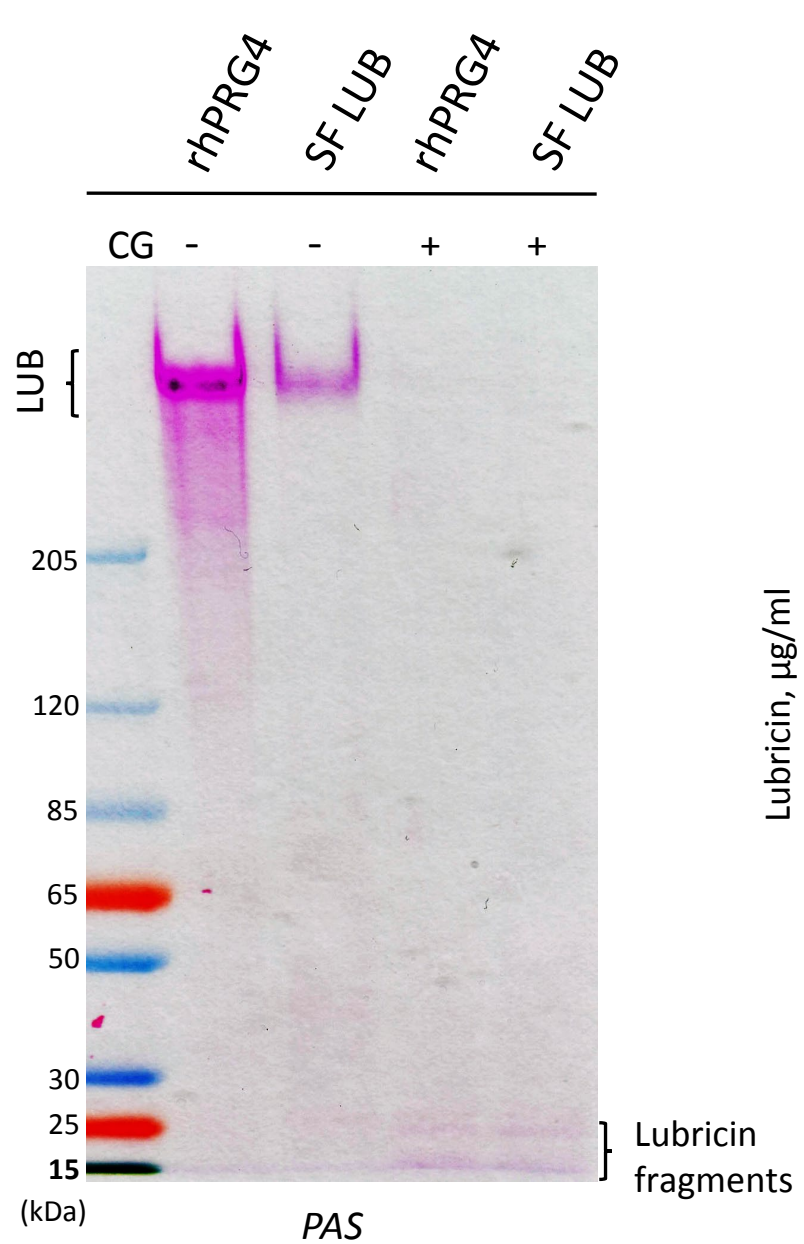

b

C

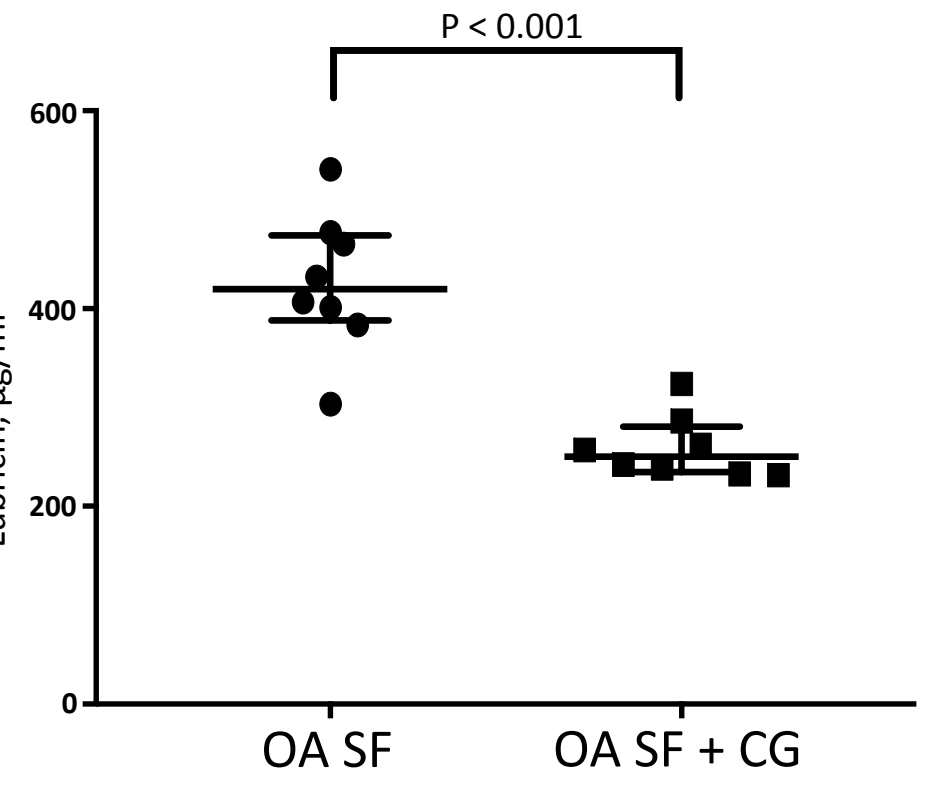

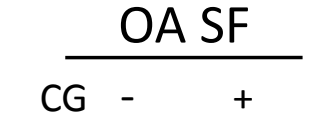

$\stackrel{\varrho}{3}$

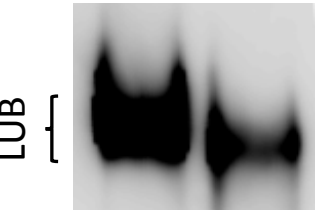

205

85

50

25
(kDa)

PNA lectin 


\section{Figure 5}

a

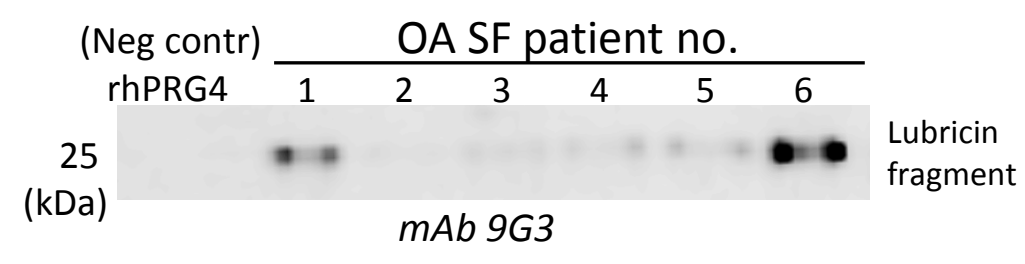

b

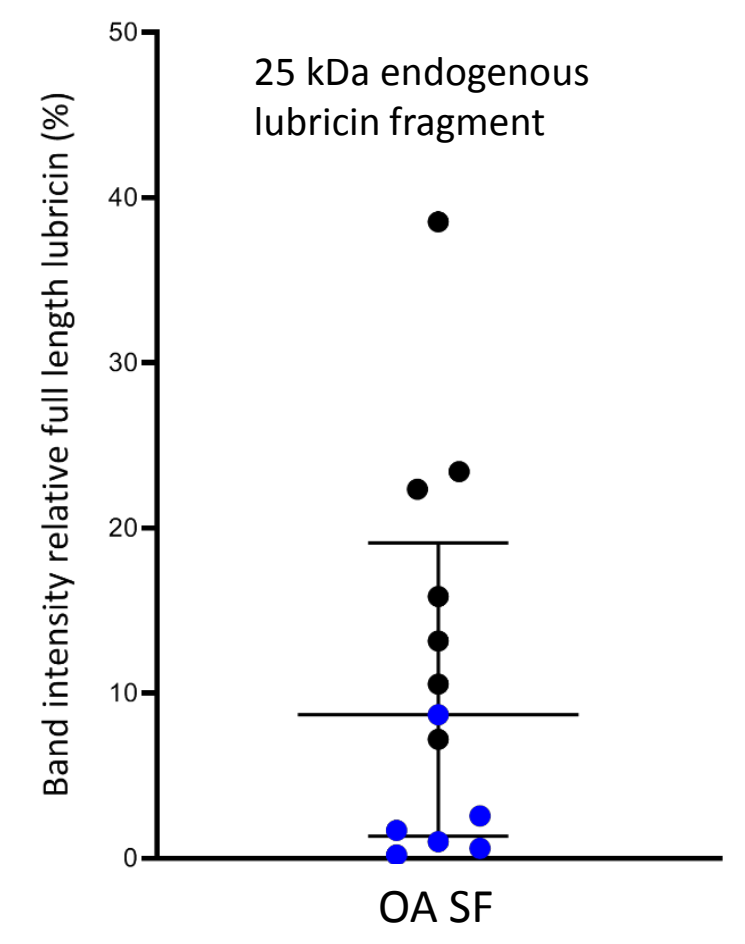

\section{C}

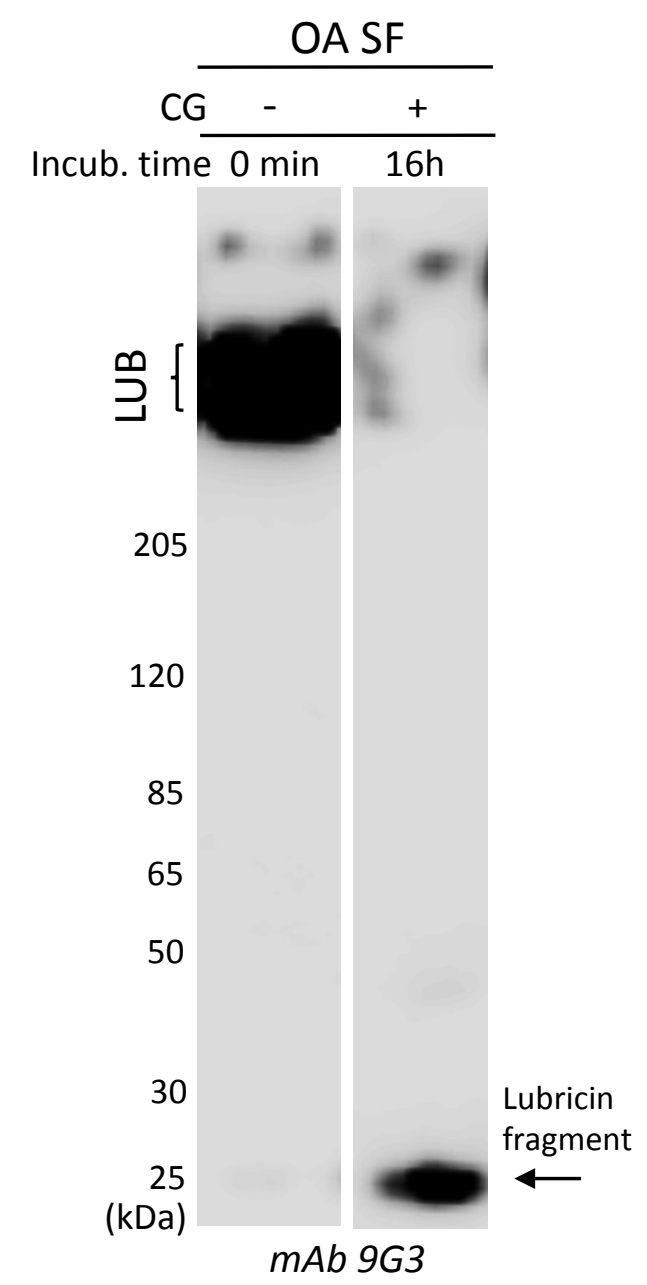


a

N-terminal

Mucin domain

C-terminal

aa 1-231 (26 kDa)

approx. 250 kDa

aa 1057-1404 (39 kDa)
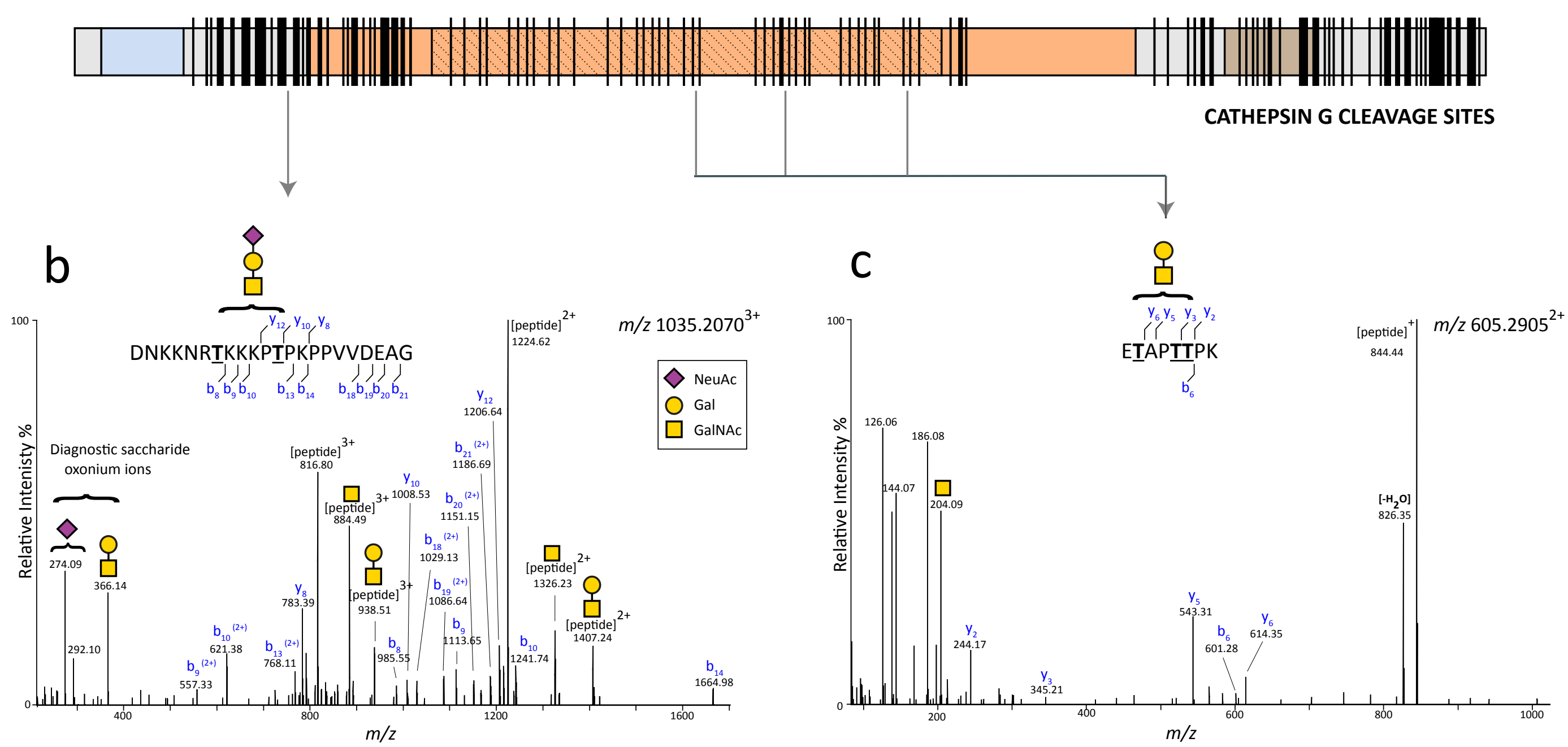

CATHEPSIN G CLEAVAGE SITES

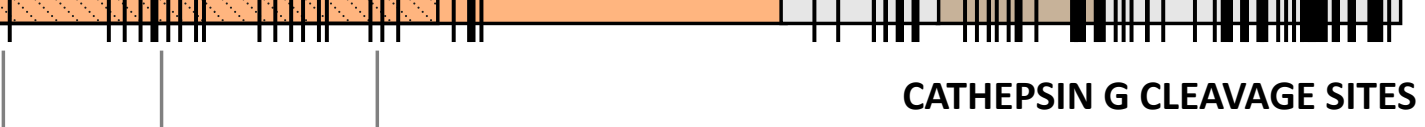

